\title{
Discriminant Phase Component for Face Recognition
}

\author{
Naser Zaeri \\ Faculty of Computer Studies, Arab Open University, P.O. Box 3322, Safat 13033, Kuwait \\ Correspondence should be addressed to Naser Zaeri, n.zaeri@aou.edu.kw \\ Received 1 September 2011; Revised 14 December 2011; Accepted 14 December 2011 \\ Academic Editor: Somaya Al-Maadeed \\ Copyright ( 2012 Naser Zaeri. This is an open access article distributed under the Creative Commons Attribution License, which \\ permits unrestricted use, distribution, and reproduction in any medium, provided the original work is properly cited. \begin{abstract}
current algorithms for face recognition involve considerable amount of computations and hence they cannot be used on devices constrained with limited speed and memory. In this paper, we propose a novel solution for efficient face recognition problem for systems that utilize small memory capacities and demand fast performance. The new technique divides the face images into components and finds the discriminant phases of the Fourier transform of these components automatically using the sequential floating forward search method. A thorough study and comprehensive experiments relating time consumption versus system performance are applied to benchmark face image databases. Finally, the proposed technique is compared with other known methods and evaluated through the recognition rate and the computational time, where we achieve a recognition rate of $98.5 \%$ with computational time of 6.4 minutes for a database consisting of 2360 images.
\end{abstract} \\ Numerous face recognition techniques have been developed owing to the growing number of real-world applications. Most of
}

\section{Introduction}

Increased security demands of modern society have augmented the importance of automatic personal identification and authentication by means of biometrics. Face recognition, among all the biometrics, is one of the most common methods that humans use in their visual interaction and perception [1]. It has received extensive attention because of its significant commercial and noncommercial applications, ranging from biometrics, information theory, law enforcement and surveillance to smart cards and access control [2]. Moreover, in video processing and Internet applications, face image retrieval is an important factor for identification and verification purposes.

On the other hand, with the wide spread of commercial portable devices, the need for more practical, costeffective, and low-power implementation of real-time face recognition systems has increased. Mobile devices such as cellular phones, personal digital assistant (PDAs), or laptops which are equipped with web browsers and mail software have become popular tools to access the Internet for both personal and business use [3-9]. Typical face recognition algorithms that found great applications in different fields (banks and airports) may not be executable in devices that are memory constrained, with computer processing unit (CPU) speed of no more than 100-500 MHz [5]. The limited memory of these CPUs put more burdens on the practical implementations of face recognition algorithms. Further, transmission and receiving of digital information from and to wireless devices are becoming very exciting due to recent developments. With the arrival of the IMT-2000 communication service, the wireless network bandwidth is rapidly widening, where the first implementation stage of this service offers broadband packet switched wireless channels of up to $384 \mathrm{kbps}$. Thus face recognition will find wide applications in this service which enables the delivery of high-quality audiovisual (AV) content to mobile users in the near future [10]. The explosive development of mobile communications, the ever-increasing importance of video communication, and the rapid development in electronics and communication engineering as well as the hardware technology strongly motivate researchers to look for techniques of low dimensionality that utilize memories of small capacities.

In this paper, we propose a practical method that can be launched on small and portable devices, where most of the current typical face recognition algorithms may not be 
executable in memory-constrained devices. The proposed method uses the phase part of the Fourier transform of face image components as the input for the classification system. To the best of our knowledge, no work has been done or presented on the frequency domain at the component level for face recognition. In [11], we have discussed the feasibility of the idea and presented a general evaluation and preliminary results of the proposed method. Here in this paper, we present a detailed study and a comprehensive description of the method, where we demonstrate a deep evaluation and analysis to show the effectiveness of implementing it in systems utilizing small memory devices. The proposed method achieves better results for recognition and huge savings in computational time when compared to other wellknown methods. Extensive experimental results of testing the proposed method are presented on four benchmark face image databases.

The organisation of the paper is as follows. A literature review that discusses component-based face recognition techniques is given in Section 2. Section 3 discusses the new proposed method for face recognition, the discriminant phase component. The experimental results and analysis are given in Section 4. Finally, concluding remarks are given in Section 5.

\section{Literature Review}

Component-based matching methods that use local features of a face image for representation have attracted lots of interest in the last few years [12-27]. After finding the local features of the face image using a set of characteristics, the local features are combined at a second stage to decide whether the input face image belongs to a given class. One of the main objectives of the component-based approach is to find the best set of components, including their locations, their numbers, and their sizes that can classify and identify the face image correctly.

Brunelli and Paggio [28], Moghaddam and Pentland [29], and Wiskott et al. [30] had early efforts in investigating the component-based face recognition. Chen et al. [22] used the boosting approach for component learned by local nonnegative matrix factorization. Ullman et al. [15] used both whole face images at an intermediate resolution and local regions at a high resolution for face verification, where components of various sizes were cropped at random locations of the images. Heisele and Koshizen [24] introduced an algorithm that learns rectangular facial components around preselected points on the face, and each component is grown iteratively. Heisele et al. in [27] restricted the location of the components to be within a predefined search region, where they added the position of the detected pre-defined components as an additional input to the classifier. The linear and second-degree polynomial support vector machines (SVMs) classifiers that were used made the system computationally expensive. Kim et al. [12] applied component-based representation for MPEG-7 face description. However, in their study no explanation or criteria were given to describe the selection process of the components.
Tan and Chen implemented adaptively weighted subpattern PCA on different parts of the face [31]. Short et al. [32] compared the performance of different photometric normalization techniques of varying complexity on components containing salient features of a face. In [33], Lin et al. proposed a multiregion approach for improving the robustness to facial expressions. Intuitively, they argued that smaller facial regions, if judiciously selected, would be less sensitive to expression variations and may lead to better overall performance. Tsai et al. [34] dealt with the impact of facial expression changes as individuals deform/express their faces either naturally or deliberately in a real-time face recognition system. Zhang et al. [35] proposed an object descriptor called histogram of Gabor phase pattern for face recognition. Their technique is based on extending quadrant-bit codes based on the Gabor transformation. Although the technique achieved high performance, it has one major drawback that lies in the high-dimensional histogram features. Zou et al. [36] reviewed and compared between some methods for face recognition through localized matching. Based on the results of the comparative experiments, they have implemented a local matching face recognition system by integrating what they have called "the best option in each step."

Tongzhou et al. [37] divided each original image sample into a certain number of subimages where all the training subimages from the same position constructed series of new training subpattern sets. Then, principal component analysis (PCA) followed by Gabor wavelet were used to extract local projection subfeature vectors to obtain a set of projection subspaces. Du and Ward [38] proposed a method that employs nonuniform multilevel selection of Gabor features. It is based on the local statistics of the Gabor features and implemented using a coarse-to-fine hierarchical strategy. Kam-Art et al. [39] proposed a method for feature extraction based on descriptive statistics of components of a face image: eyes, nose, and mouth. Hua and Liu [40] proposed a method for facial expression recognition based on local feature bidirectional two-dimensional PCA. Also, De Marsico et al. [41] presented a technique based on partitioned iterated function by working on each face component independently.

Although many works have aimed at developing robust face recognition algorithms, relatively few works have focused on adapting the technology to the domain of mobile computing and memory saving. This may be explained by the fact that mobile technology did not find interesting applications in our lives before a few years ago, where the advancement in the mobile industry and wireless communication system design have reached a matured level. Harguess and Aggarwal [42] have demonstrated the effectiveness of using the average-half-face as an input to face recognition algorithms for an increase in accuracy and a potential decrease in storage and computation time. Zaeri et al. [43] have applied component-based linear discriminant analysis approach to systems requiring high-speed performance. Their proposed method enhanced the performance of the system and achieved high recognition rates.

Choi et al. [44] Proposed incremental two-directional two-dimensional PCA for real-time face recognition in embedded system $\left(\mathrm{I}(2 \mathrm{D})^{2} \mathrm{PCA}\right)$. They have combined 
the advantages of both the incremental principal component analysis (IPCA) method and the (2D) ${ }^{2} \mathrm{PCA}$ method. The $(2 \mathrm{D})^{2}$ PCA method is faster than the conventional PCA because the resulted covariance matrix size is smaller in the case of $(2 \mathrm{D})^{2}$ PCA. From a memory point of view, the IPCA is more efficient than the conventional PCA since the IPCA can update the current eigenvector using only the new data and the previous eigenvector without considering the whole data. Their method was tested on only the ORL and Yale databases, where the best obtained success rate was around $86 \%$ for the ORL database. Atta and Ghanbari [45] proposed a method for low memory requirements that is based on extraction of features with the discrete cosine transform (DCT) pyramid, in contrast to the conventional method of wavelet decomposition. The DCT pyramid performed on each face image decomposes it into an "approximation subband" and "reversed blocks" that contain the high frequency coefficients of the DCT pyramid. A set of block-based statistical measures was calculated from the DCT pyramid subbands to reduce the dimensionality of the feature vectors. Their experiments were conducted on the ORL and FERET databases, where the facial portion of each image was resized to $80 \times 80$. The best obtained result was around $90 \%$ and less than $96 \%$, for the FERET and the ORL databases, respectively.

He et al. [46] suggested a hardware implementation for face image acquisition and recognition system based on SOPC platform, which takes the advantage SOPC (system-on-a-programmable-chip) technology. SOPC is a programmable system with a flexible design that is expandable and upgradeable where the main logical functions of the whole system are completed by a single chip. Sisodia et al. [47] tried to reduce the large training time and memory consumption using incremental support vector machine (ISVM). The performance of their system was tested on the ORL database only. Xi et al. [48] developed a hierarchical correlation based face verification (HCFV) scheme for devices with memory size of $1 \mathrm{MB}$. Their technique is based on analyzing the relations among multiple cross-correlation peaks which are generated from selected subregions of a face. In addition, global information was used in the scheme to smooth out the error that occurred in the correlation of the feature segments. The proposed scheme has achieved better performance than the conventional correlation-based schemes when applied to Yale face database. Vazquez-Fernandez et al. [49] proposed a simple face recognition algorithm for implementation in mobile devices based on local binary patterns (LBP) features. The algorithm generates a photo sharing application that allows users to link biometric contact information automatically to faces. The implementation was made for the Google Android platform, and was tested on a local face image database consisting of 50 persons each represented by 4 images. Wang and Cheng [50] explored the strategies of mapping a computer vision application to a smartphone platform and used face recognition as the application driver. Their study aimed at minimizing the energy and optimizing the performance, as the power and energy consumption is the most critical design consideration for smartphone applications.
They have demonstrated that there is a wide range of available tradeoffs among performance, energy consumption, and accuracy.

\section{Discriminant Phase Component}

Typical images share certain features which, when regarded as points in $\mathfrak{R}^{2}$ space, translate to a group geometry subjected to quick approximation by several linear schemes. Mathematically, since the face images take place in linear spaces, we will represent the face image components using powerful fundamental or basis images that can be effectively used to describe detailed levels within large classes of images (or components of these images). A given image is resolved into a linear combination of the basis images and by examining the coefficients' weight, the importance of particular details to the entire given image is realized. We transform the image components into the frequency domain where the important and discriminant features are distinguished, and hence the recognition and identification processes are made based on the components' new shape.

It is well known that the Fourier transform $\hat{x}$ of a 1D signal $x$ is a complex-valued vector (or a matrix for a $2 \mathrm{D}$ image) containing the amplitudes and the phases of the fundamental frequencies that make up $x$. Each coefficient of $\hat{x}$ indicates the strength of a particular frequency in $x$. The Fourier transform gives us a direct control over these frequencies, for example, replacing an entry in $\hat{x}$ with zero "removes" the corresponding frequency from $x$. Coefficients in $\hat{x}$ of small magnitude indicate frequencies with weak mathematical presence in $x$, and discarding them may be done with relative impunity. In applications where memory and computational complexity are of great concern, discarding these coefficients offer great advantages. It was shown in $[51,52]$ that the phase angle of the Fourier transform retains most of the information about the image. The phase spectrum acts to position the bright and dark spots in the image in order to form regions that are recognizable by a viewer.

As such, and as the phase information retains the most of the intelligibility features of the image, the image variation will be modelled by keeping the phase spectrum of the image only. Also, since much of the noise, distortion, and image corruption are noticeable in the magnitude part of the image spectrum, then taking off the magnitude part will reduce the effect of the weak features and will keep the discriminative features that are presented in the phase part of the Fourier transform.

Figure 1 shows the effect of both the magnitude and the phase parts of the Fourier transform for one component of a face image, the eyes. Figure 1(b) is obtained by computing the inverse Fourier transform of Figure 1(a) using the phase information only. Note that the key shape features of this face component are unmistakably from Figure 1(a). Figure 1(c) is obtained by computing the inverse Fourier transform of Figure 1(a) using the magnitude part only. Note that it contains only intensity information, with the dc term being the most dominant (in the corner). Figure 2 shows 


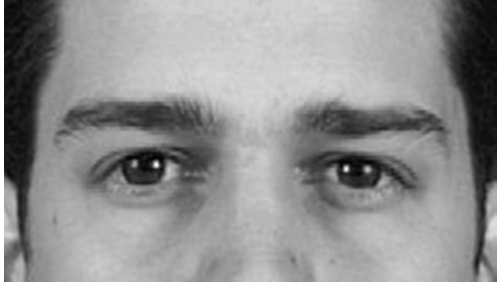

(a)

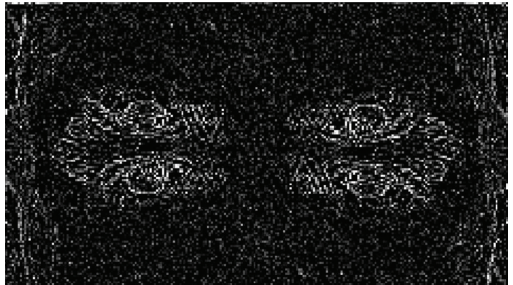

(b)

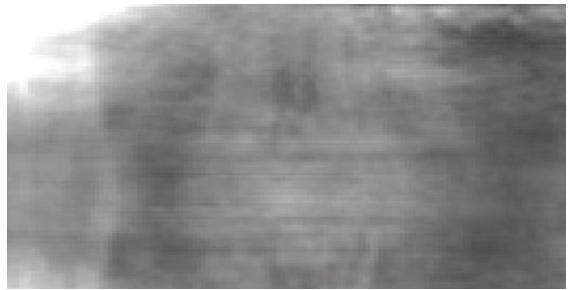

(c)

Figure 1: (a) The eye component, (b) and (c) the phase and the magnitude parts of the Fourier transform of the eyes.

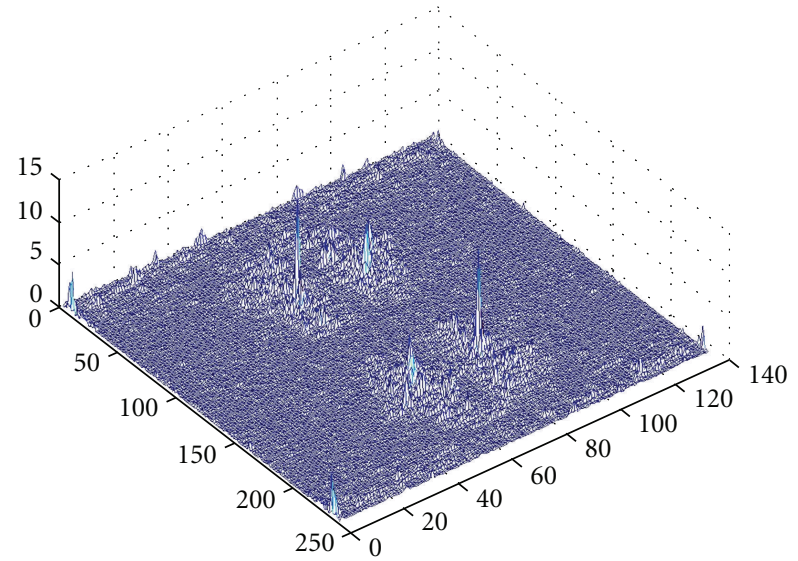

(a)

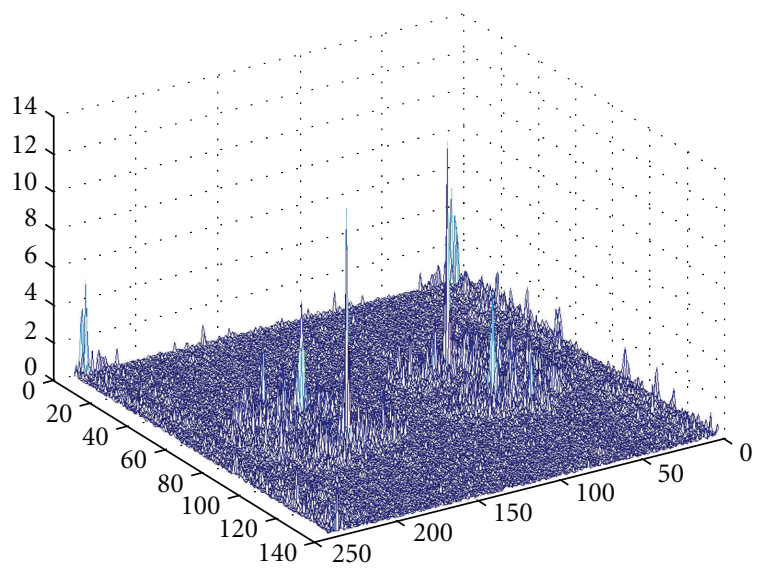

(c)

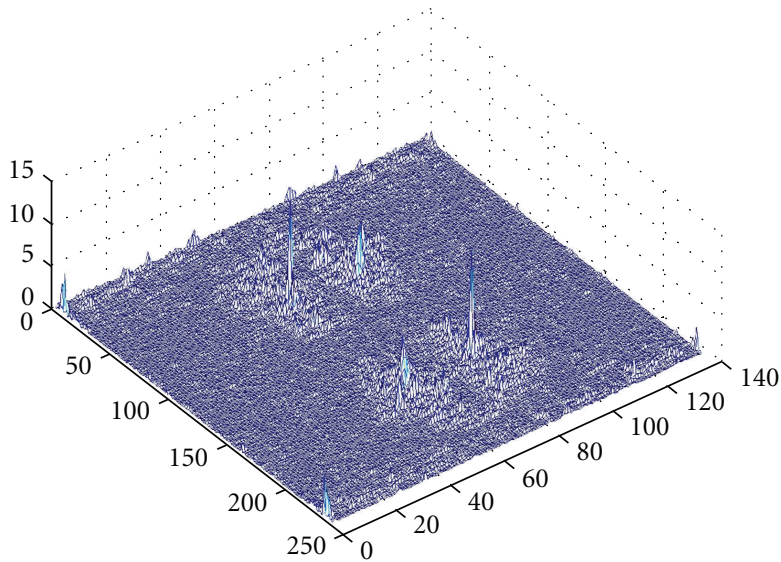

(b)

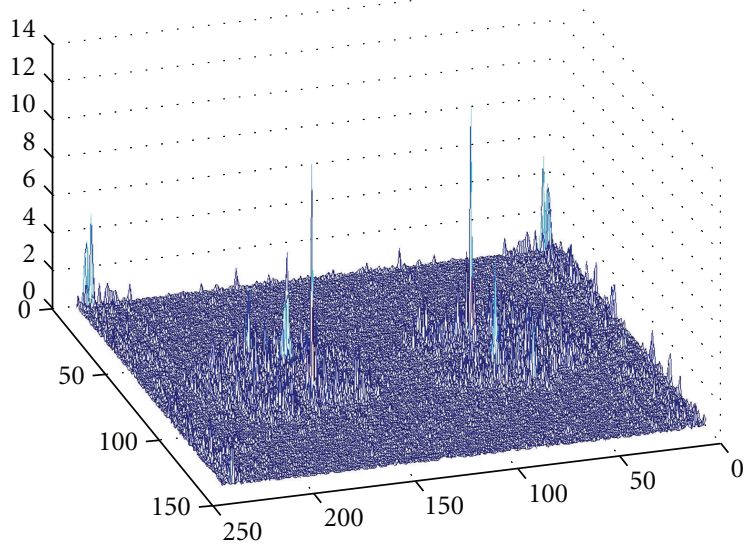

(d)

Figure 2: Four different examples of discriminant phases for different eyes components.

a 3D plot of the absolute value of the phase angles of the Fourier transform for four different eyes components. Note that the eyes discriminant features are preserved and can be clearly distinguished from the rest of the area surrounding it.

We will consider the local components of a face image (instead of the entire face image) for recognition, as it is expected to achieve higher correct recognition rates since the image variations on the component level is limited when compared to the variations within the entire image. Then, the linear discriminant analysis (LDA) method is applied to the phase components. The LDA-based techniques have been extensively used in face recognition due to its optimal performance and time-efficient matching for multiclass face recognition [34, 53-58]. Classical discriminant analysis aims at deriving a transformation such that the transformed data have maximum separation between classes and minimum separation within classes. Hence, LDA emphasizes variations among different classes while the variations of the same class are deemphasized, and consequently it finds a direction on which data are well classwise clustered. Figure 3 details the proposed technique. 
The first step is to partition the face image into $(L)$ components. Selecting the right size of a component is a difficult task and could depend on many factors such as the number of images in the database, the size of these images, the variations in the input images, the required identification speed, and the required recognition rate. These factors influence the decision of choosing the best component size, number, and location, which are not independent. The size and the number of these distinguished components are adapted and optimized by an algorithm that automatically finds the best presentation of the face image and consequently the best performance. These components are found using the sequential floating forward search (SFFS) algorithm [59], which will be explained in the next subsection.

As shown in Figure 3, each one of the $L$ components of the image undergoes a Fourier transform. Then the phase angle is extracted, where the database of images are represented by phase spectrum of the Fourier transform. Then each of the " $L$ " phase components undergoes LDA transformations (PCLDA). In a similar way to the training images, the same procedure is applied to the test (query) image. Now, for every component of the test image we find the distance between its PCLDA transformation and the corresponding training PCLDA transformation. This is mathematically expressed in the next subsection.

3.1. Mathematical Representation of the System. Assume that a data matrix $\chi=\left\{x_{1}, x_{2}, \ldots, x_{M}\right\} \in \mathfrak{R}^{N \times M}$ is given, where $x_{i} \in \mathfrak{R}^{N}$ is an $N$-dimensional column vector representing the face image and $M$ is the number of training images. Each vector belongs to one of $C$ object classes $\left\{\chi_{1}, \chi_{2}, \ldots, \chi_{C}\right\}$. Classical discriminant analysis aims at deriving a transformation $W \in$ $\mathfrak{R}^{N \times n}(n \leq N)$ which maps a vector $x$ to $y=W^{T} x, y \in \mathfrak{R}^{n}$ such that the transformed data have maximum separation between classes and minimum separation within classes. The between-class $S_{B}$ and within-class $S_{W}$ scatter matrices in LDA for the $c$-class case are given, respectively, by

$$
\begin{gathered}
S_{B}=\sum_{i=1}^{C} n_{i}\left(m_{i}-m\right)\left(m_{i}-m\right)^{T}, \\
S_{W}=\sum_{i=1}^{C} \sum_{x \in \chi_{c}}\left(x-m_{i}\right)\left(x-m_{i}\right)^{T},
\end{gathered}
$$

where $m_{i}$ denotes the class mean, $m$ is the global mean of the entire sample set, and $n_{i}$ denotes the number of samples in class $c$. Given the between-class scatter matrix $S_{B}$ and the within class scatter matrix $S_{W}$ for the face space, the attempt is to solve the generalised eigenvalue problem

$$
S_{B} u_{i}=\lambda_{i} S_{W} u_{i} .
$$

In our proposed technique, and as we have explained earlier, all the images are first partitioned into $L$ facial components. After that, the Fourier transform is applied to each component $x(j, k)$ of a face image:

$$
\hat{x}(u, v)=\frac{1}{N} \sum_{j, k=0}^{N-1} x(j, k) e^{-2 \pi i(j u-k v) / N},
$$

for any pairs of integers $u$ and $v$ or $j$ and $k$. The images (patches) of each component are represented in a vector form with the $k$ th component being denoted as $\left\{c_{1}^{k}, \ldots, c_{M}^{k}\right\}$. Next, the phase angle for the $k$ th facial component is extracted, and then the corresponding LDA transformation matrix $W^{k}$ is computed. As shown in Figure 3, during testing, the $L$ vectors $\left\{c^{1}, \ldots, c^{L}\right\}$ that correspond to the facial component patches are extracted from a face image $x_{i}$ of the test data set. Next, a set of LDA feature vectors $y=\left\{y^{1}, \ldots, y^{L}\right\}$ is extracted from the test image $x_{i}$ using the corresponding LDA transformation matrices as

$$
y^{k}=\left(W^{k}\right)^{T} c^{k}, \quad k=1, \ldots, L .
$$

In this paper, the nearest neighbour classifier with Euclidean distance was used for classification. The Euclidean distance is used to measure the distances from the probed feature vector to the reference feature vectors in the gallery. The lower the distance is the closer the two vectors become. For a feature vector $V$, the Euclidean distance is defined as

$$
D=\sqrt{\sum_{z=1}^{d}\left(V_{\text {training }, z}-V_{\text {testing, } z}\right)^{2}},
$$

where $d$ is the dimension of the feature vector. Figure 4 shows an example of the resultant between-class-scatter-matrix $S_{B}$ and within-class-scatter-matrix $S_{W}$ for the phase spectrum of the Fourier transform for one component of a face image database.

At this stage, we have a candidate for each component that represents an identity of the test image. Next, we find the maximum number of components scoring the same candidate (identity) and hence, recognising the test image of that person. This is basically a fusion process where we fuse the information coming from each component to produce a single score that represents the combined decisions of the components result. The fusion method that is used is the Voting method, which outputs a score equal to the number of component classifiers that output scores above their respective thresholds. For example, if we have five classifiers and three of these classifiers give a score belonging to identity $A$, while the fourth classifier suggests identity $B$ and the fifth classifier gives a result in favour of identity $C$, then based on the Voting method the system decides that the image belongs to identity $A$. It should be noted that the study of the fusion techniques is beyond the scope of this paper. As such, we will not be investigating the best fusion technique to be used.

To find the best subset from a set of features, the SFFS search method is used which provides a very good performance when compared to other suboptimal search methods and it is computationally much less expensive than the optimal methods [59]. The proposed scheme shown in Figure 3 uses the SFFS algorithm to find the best location of a certain number of components, which can give the highest possible recognition rate. In this regard, the SFFS method includes new best features (here the feature is the phase component) that when added to the current feature set, 


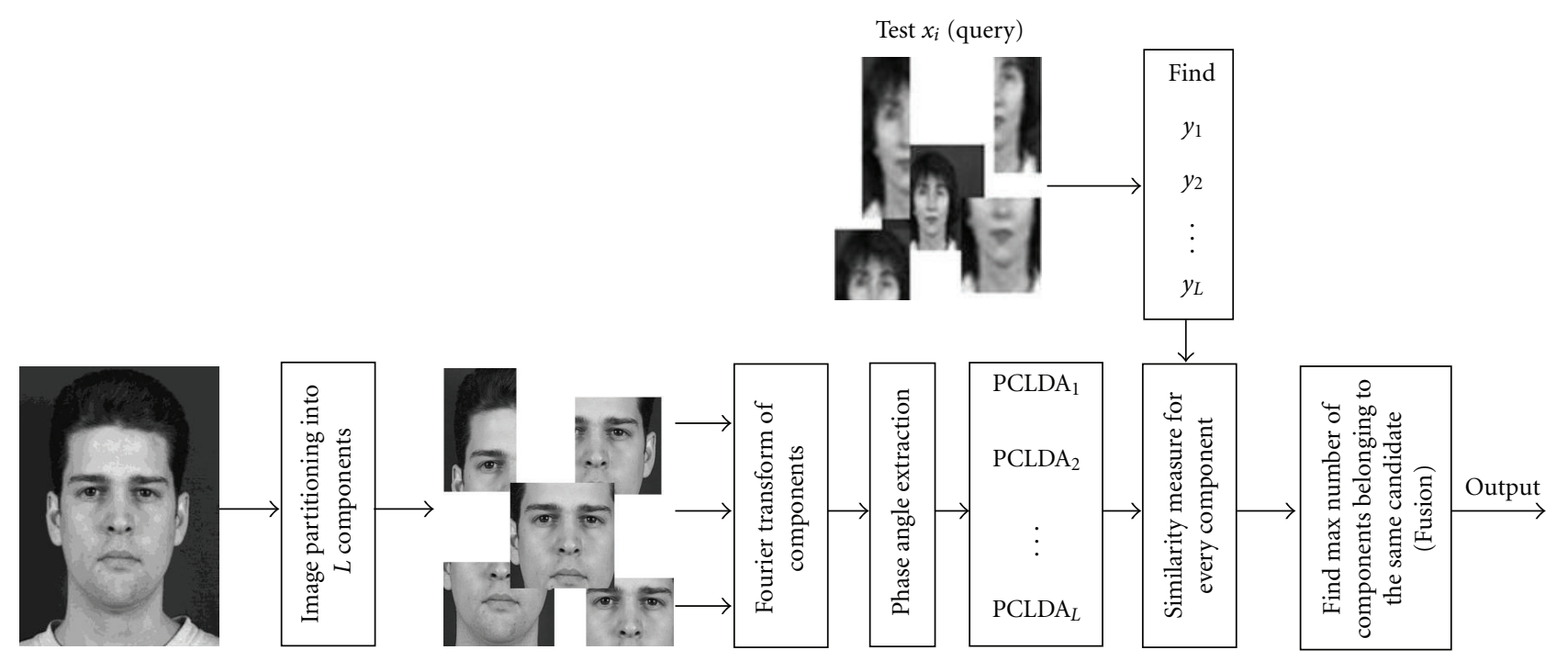

FIgURE 3: The new proposed technique: discriminant phase component.

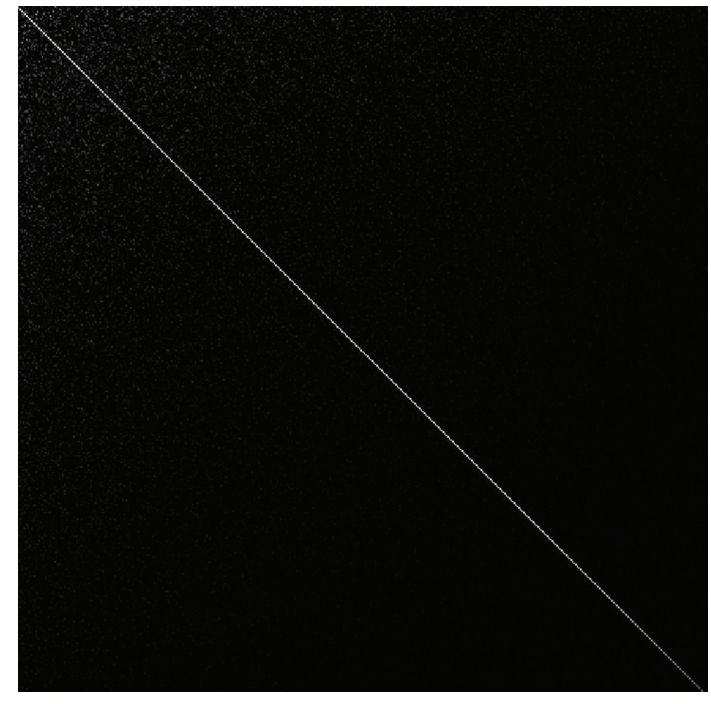

(a)

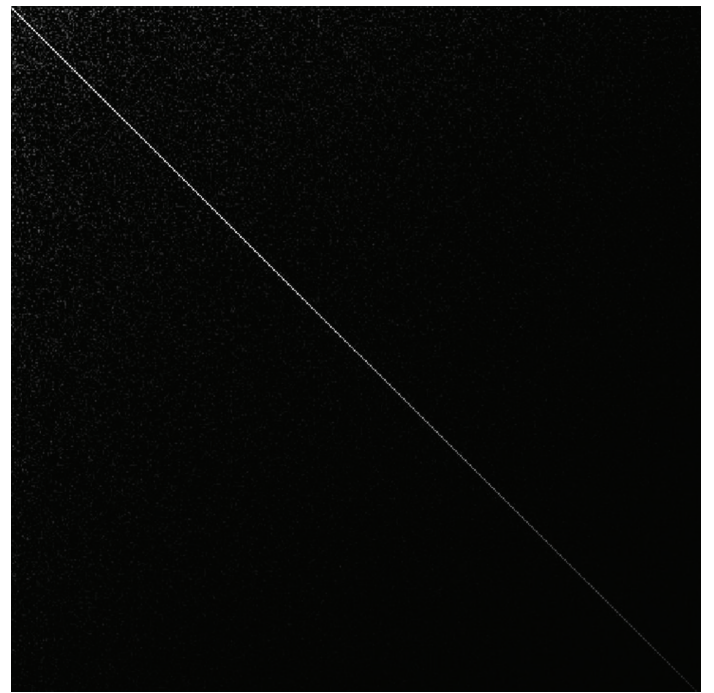

(b)

FIGURE 4: An example of the resultant between-class-scatter-matrix (on the left) and within-class-scatter-matrix (on the right) for a phase spectrum of a component face image.

the error rate is minimized. In addition, the SFFS algorithm excludes the worst feature during the selection process in order to further improve the selection of the feature set. Briefly, the SFFS method can be explained as follows.

Suppose $k$ features have already been selected from the complete set of measurements $Y=\left\{y_{j} \mid j=1,2, \ldots, D\right\}$ of $D$ available features to form the set $X_{k}=\left\{x_{i}: 1 \leq i \leq k, x_{i} \in\right.$ $Y\}$ with the corresponding criterion function $J\left(X_{k}\right)$, which represents the error rate. In addition, the values of $J\left(X_{i}\right)$ for all preceding subsets of size $i=1,2, \ldots, k-1$, are known and stored.

Step 1. Select feature $x_{k+1}$ from the set of available measurements, $Y-X_{k}$ to form feature set $X_{k+1}$ such that $J\left(X_{k}+x_{k+1}\right)$ is minimised with respect to $x_{k+1}$.
TABLE 1: The results of 4-component size $30 \times 30$ for the new method.

\begin{tabular}{lcc}
\hline & Rank 1 result (\%) & Time $(\mathrm{min})$ \\
\hline ORL & 99.9 & 4.6 \\
xm2vts (C1) & 99.4 & 20.5 \\
xm2vts (C2) & 99.7 & 20.9 \\
FERET (Fb) & 99.5 & 23.3 \\
FERET (Fc) & 97.9 & \\
Yale & 98.2 & 2.1 \\
\hline
\end{tabular}

Step 2. Find a feature, $\hat{x}_{k+1}$, in $X_{k+1}$ the removal of which will minimise $J\left(X_{k+1}-\hat{x}_{k+1}\right)$ with respect to $\hat{x}_{k+1}$. 
TABle 2: The results of 4 -component size $25 \times 25$ for the new method.

\begin{tabular}{lcc}
\hline & Rank 1 result (\%) & Time (min) \\
\hline ORL & 99.5 & 3.7 \\
xm2vts (C1) & 99.1 & 16.3 \\
xm2vts (C2) & 99.6 & 16.4 \\
FERET (Fb) & 99.2 & 19.1 \\
FERET (Fc) & 97.4 & \\
Yale & 97.8 & 1.7 \\
\hline
\end{tabular}

TABle 3: The results of 4 -component size $20 \times 20$ for the new method.

\begin{tabular}{lc}
\hline & Rank 1 rate \\
\hline ORL & 99.4 \\
xm2vts (C1) & 98.5 \\
xm2vts (C2) & 99 \\
FERET (Fb) & 97.9 \\
FERET (Fc) & 95.4 \\
Yale & 94.1 \\
\hline
\end{tabular}

TABLE 4: The results of 4 -component size $15 \times 15$ for the new method.

\begin{tabular}{lc}
\hline & Rank 1 rate \\
\hline ORL & 66.2 \\
xm2vts (C1) & 64.2 \\
xm2vts (C2) & 65 \\
FERET (Fb) & 63.1 \\
FERET (Fc) & 62.2 \\
Yale & 60.4 \\
\hline
\end{tabular}

Step 3. If $x_{k+1}=\hat{x}_{k+1}$, then $k=k+1$ and return to Step 1 .

Step 4. If $x_{k+1} \neq \hat{x}_{k+1}$, then $\hat{X}_{k}=X_{k+1}-\hat{x}_{k+1}$.

Step 5. If $k=2$, then $X_{k}=\hat{X}_{k}$, and return to Step 1 .

Step 6. Find a feature, $\hat{x}_{r}$, in $\hat{X}_{k}$, the removal of which will minimise $J\left(\hat{X}_{k}-\hat{x}_{r}\right)$.

Step 7. If $J\left(\hat{X}_{k}-\hat{x}_{r}\right)>J\left(X_{k-1}\right)$, then $X_{k}=\hat{X}_{k}$, and return to Step 1.

Step 8. If $J\left(\hat{X}_{k}-\hat{x}_{r}\right) \leq J\left(X_{k-1}\right)$, then $X_{k-1}=\hat{X}_{k}-\hat{x}_{s}, k=k-1$.

Step 9. If $k=2$ return to Step 1, otherwise return to Step 6.

\section{Experimental Results and Analysis}

We have carried out experiments on four independent and different databases; namely, the ORL, xm2vts, FERET, and Yale databases. The ORL set includes 400 images of 40 different individuals where each individual is represented by 10 . The system was trained using 5 images for each person from this set. The xm2vts set have 2360 images for 295 different individuals with each individual represented by 8 different images taken at four different sessions, with two shots at each session. The xm2vts uses a standard protocol, referred to as Lausanne protocol [60]. According to Lausanne protocol, the database is split into three groups: the training group, evaluation group, and the testing group. There are two configurations of the xm2vts database. In the first configuration $(\mathrm{C} 1)$, the client images for training and evaluation were collected from each of the first three sessions. In the second configuration (C2), the client images for training were collected from the first two sessions and the client images for evaluation from the third.

In the FERET database, all frontal face pictures are divided into five categories: $\mathrm{Fa}, \mathrm{Fb}, \mathrm{Fc}$, Dup1, and Dup2. In the FERET tests, $1196 \mathrm{Fa}$ pictures are gallery samples and there is only one image per person in the gallery. Therefore, the size of the gallery, 1196, indicates the number of classes. $1195 \mathrm{Fb}$ and $194 \mathrm{Fc}$ pictures are named as $\mathrm{Fb}$ and $\mathrm{Fc}$ probes, respectively. In our experiments, we have utilized the $\mathrm{Fb}$ and $\mathrm{Fc}$ categories. $\mathrm{Fb}$ pictures were taken at the same day as Fa pictures and with the same camera and illumination condition. Fc pictures were taken at the same day as $\mathrm{Fa}$ pictures but with different cameras and illumination. The cumulative match curve is used in the FERET tests to compare the performance of different algorithms. The usual definition of classification accuracy is the percentage of correct matches of rank 1. The Yale face database contains 165 gray scale images of 15 individuals. There are 11 images per subject, one per different facial expression or configuration. This set has considerable variations in facial expressions and illuminations.

Initially, we have tested the effect of the component size on system performance. We started with 4 components $(L=$ 4) each of size $30 \times 30$. The results are shown in Table 1 for the four different databases. Note that although a high successful recognition rate is achieved, the processing time is relatively large for the intended applications. For example, in the case of FERET $(\mathrm{Fb})$ database it took the system more than 23 minutes to attain the 99.5 classification rate.

Then, the size of the components has been reduced to $25 \times 25$. The corresponding results are shown in Table 2 , where we can see that the classification rate is still high. Further, the processing time is reduced. From Tables 1 and 2, we deduce that reducing the component size has a great effect on reducing the computational time. Eventually, the system has further reduced the component size to $20 \times 20$. Table 3 shows the results, where the high classification rate is still attainable.

To further emphasize the importance of the component size, the size was reduced to $15 \times 15$. Table 4 lists the results of the recognition rate, which has significantly dropped (best obtained result was $66.2 \%$ for the ORL database). We have conducted a few more experiments taking five and six components from the face image with component size of $15 \times 15$. The results obtained from the experiments were not attractive.

Hence, the results suggest increasing the number of components while keeping the component size at $20 \times 20$. 
TABle 5: The time consumed (in minutes) and the success recognition rate for the different number of components using the ORL database.

\begin{tabular}{lccccccc}
\hline & 4 comp.-size & 4 comp.-size & 4 comp.-size & 5 comp.-size & 6 comp.-size & 7 comp.-size & 14 comp.-size \\
& $30 \times 30$ & $25 \times 25$ & $20 \times 20$ & $20 \times 20$ & $20 \times 20$ & $20 \times 20$ & $20 \times 20$ \\
\hline Time $(\mathrm{min})$ & 4.6 & 3.7 & 1.3 & 1.7 & 2.2 & 2.9 & $\mathbf{3 . 6}$ \\
Success rate $\%$ & 99.9 & 99.5 & 99.4 & 99.5 & 99.8 & 99.9 & $\mathbf{1 0 0}$ \\
\hline
\end{tabular}

TABle 6: The time consumed (in minutes) and the success recognition rate for the different number of components using the xm2vts (C1) database.

\begin{tabular}{lccccccc}
\hline & 4 comp.-size & 4 comp.-size & 4 comp.-size & 5 comp.-size & 6 comp.-size & comp.-size & comp.-size \\
& $30 \times 30$ & $25 \times 25$ & $20 \times 20$ & $20 \times 20$ & $20 \times 20$ & $20 \times 20$ & $20 \times 20$ \\
\hline Time $(\mathrm{min})$ & 20.5 & 16.3 & 6.4 & 8.6 & 11.4 & 14.5 & $\mathbf{1 7 . 8}$ \\
Success rate $\%$ & 99.4 & 99.1 & 98.5 & 98.9 & 99.1 & 99.2 & $\mathbf{9 9 . 3}$ \\
\hline
\end{tabular}

TABLE 7: The time consumed (in minutes) and the success recognition rate for the different number of components using the xm2vts (C2) database.

\begin{tabular}{lccccccc}
\hline & 4 comp.-size & 4 comp.-size & 4 comp.-size & 5 comp.-size & 6 comp.-size & comp.-size & 14 comp.-size \\
& $30 \times 30$ & $25 \times 25$ & $20 \times 20$ & $20 \times 20$ & $20 \times 20$ & $20 \times 20$ & $20 \times 20$ \\
\hline Time $(\mathrm{min})$ & 20.9 & 16.4 & 6.6 & 8.7 & 11.2 & 14.6 & $\mathbf{1 7 . 4}$ \\
Success rate $\%$ & 99.7 & 99.6 & 99 & 99.3 & 99.6 & 99.8 & $\mathbf{9 9 . 9}$ \\
\hline
\end{tabular}

TABLE 8: The time consumed (in minutes) and the success recognition rate for the different number of components using the FERET (Fb) database.

\begin{tabular}{lccccccc}
\hline & $\begin{array}{c}4 \text { comp.-size } \\
30 \times 30\end{array}$ & $\begin{array}{c}4 \text { comp.-size } \\
25 \times 25\end{array}$ & $\begin{array}{c}4 \text { comp.-size } \\
20 \times 20\end{array}$ & $\begin{array}{c}5 \text { comp.-size } \\
20 \times 20\end{array}$ & $\begin{array}{c}6 \text { comp.-size } \\
20 \times 20\end{array}$ & $\begin{array}{c}7 \text { comp.-size } \\
20 \times 20\end{array}$ & $\begin{array}{c}14 \text { comp.-size } \\
20 \times 20\end{array}$ \\
\hline Time $(\mathrm{min})$ & 23.3 & 19.1 & 5.8 & 7.7 & 10.8 & 13.1 & $\mathbf{1 5 . 6}$ \\
Success rate $\%$ & 99.5 & 99.2 & 97.9 & 98.2 & 98.3 & 98.4 & $\mathbf{9 8 . 9}$ \\
\hline
\end{tabular}

TABLE 9: The time consumed (in minutes) and the success recognition rate for the different number of components using the Yale database.

\begin{tabular}{lccccccc}
\hline & 4 comp.-size & 4 comp.-size & 4 comp.-size & 5 comp.-size & 6 comp.-size & comp.-size & com comp.-size \\
& $30 \times 30$ & $25 \times 25$ & $20 \times 20$ & $20 \times 20$ & $20 \times 20$ & $20 \times 20$ & $20 \times 20$ \\
\hline Time $(\mathrm{min})$ & 2.1 & 1.7 & 0.85 & 0.9 & 1.1 & 1.2 & $\mathbf{1 . 6}$ \\
Success Rate $\%$ & 98.2 & 97.8 & 94.1 & 94.7 & 94.9 & 95.3 & $\mathbf{9 6 . 3}$ \\
\hline
\end{tabular}

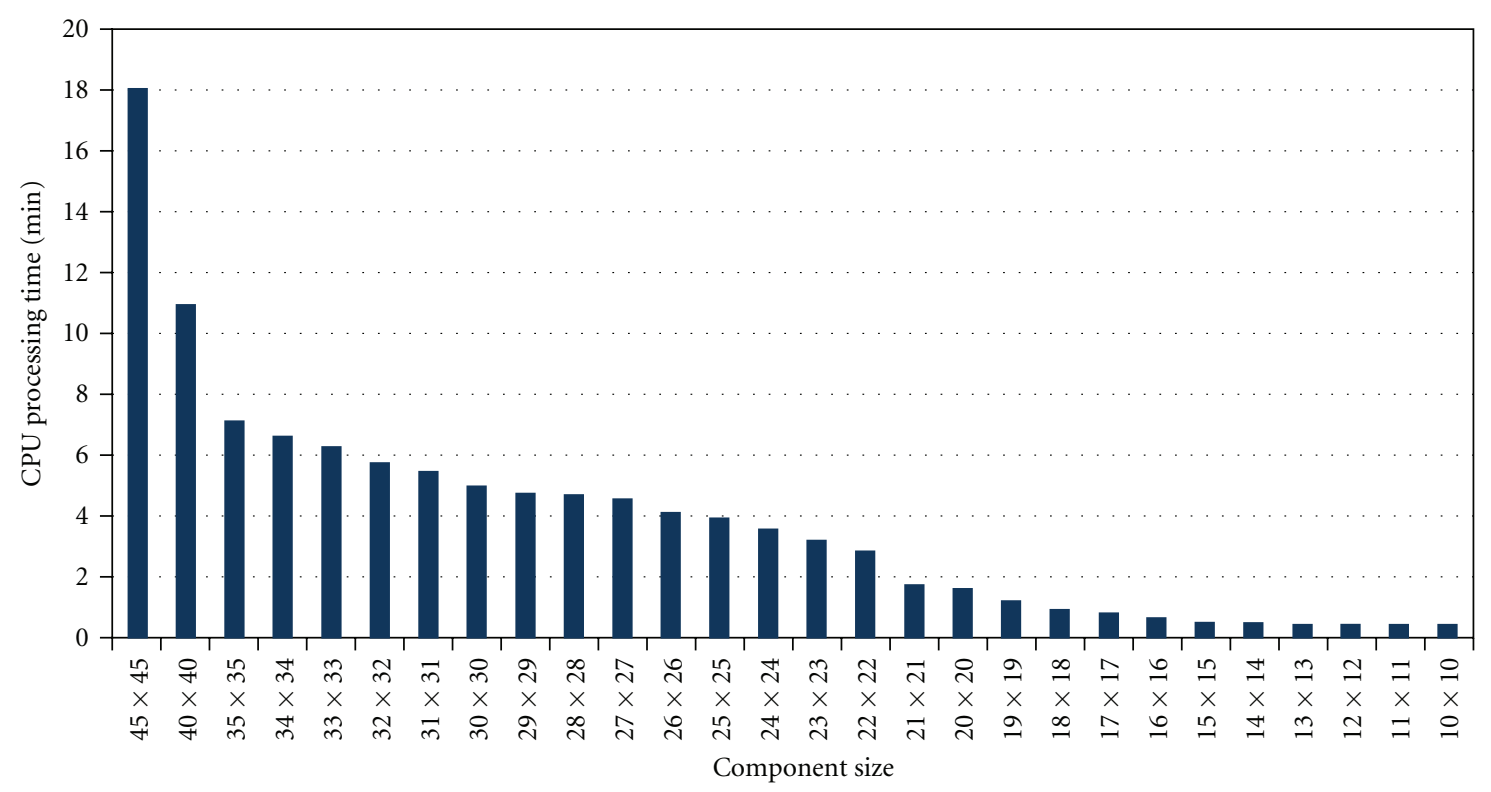

FIGURE 5: Computation time for a single block (component) for the xm2vts (C1) database case. 


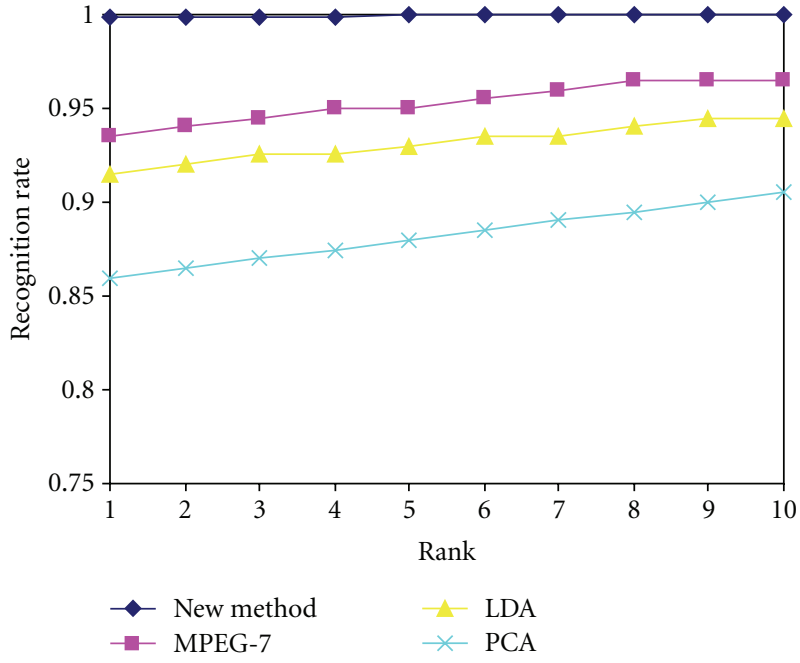

FIgURE 6: CMS results for the ORL database.

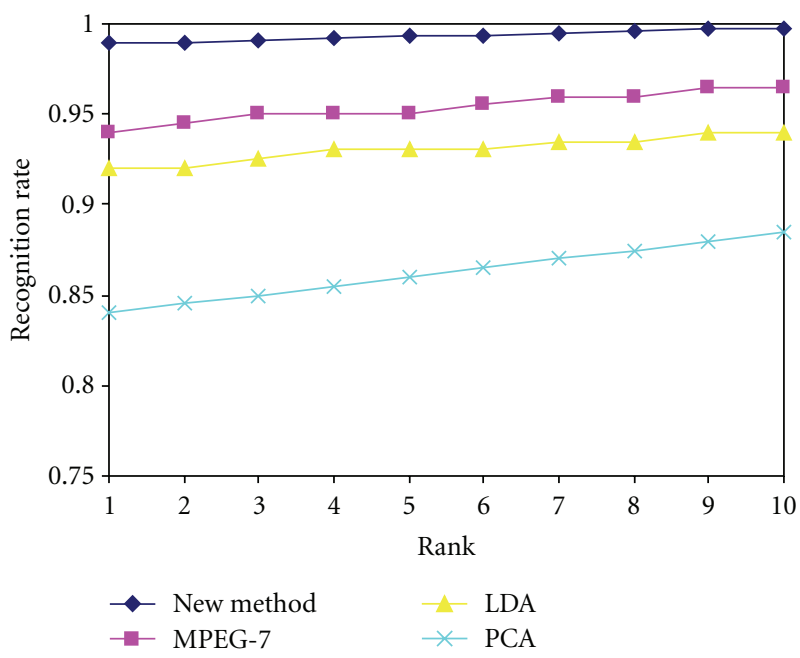

Figure 7: CMS results for the FERET (Fb) database.

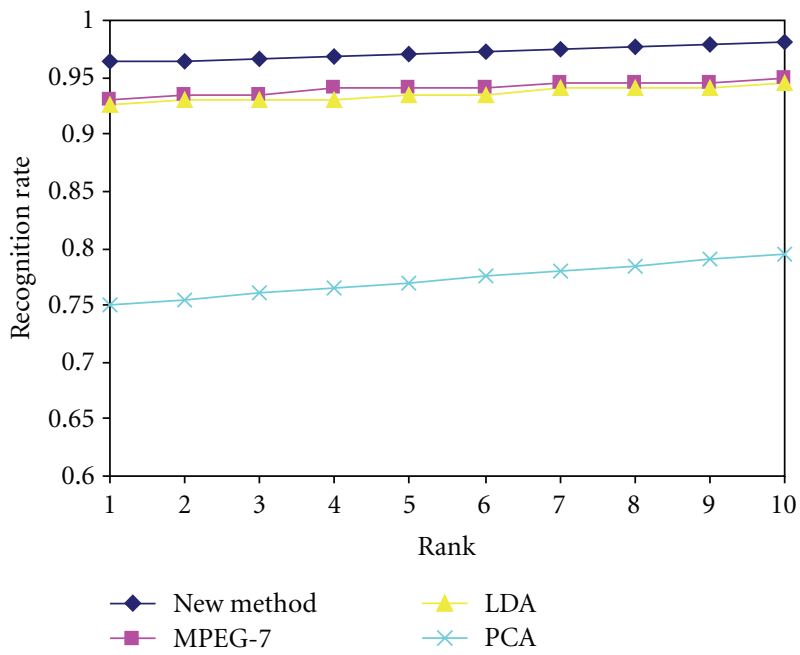

FIGURE 8: CMS results for the Yale database.
TABLE 10: The time consumed (in minutes) and the success recognition rate for the different methods using the ORL database.

\begin{tabular}{lcccc}
\hline & PCA & LDA & MPEG-7 & New method \\
\hline Time $(\min )$ & 3.84 & 9.7 & 11.8 & 3.6 \\
Success rate $\%$ & 86 & 91.5 & 93.5 & 100 \\
\hline
\end{tabular}

TABLE 11: The time consumed (in minutes) and the success recognition rate for the different methods using the xm2vts $(\mathrm{C} 1)$ database.

\begin{tabular}{lcccc}
\hline & PCA & LDA & MPEG-7 & New method \\
\hline Time $(\min )$ & 26.7 & 73.4 & 84.1 & 17.8 \\
Success rate $\%$ & 73 & 90.4 & 91.2 & 99.3 \\
\hline
\end{tabular}

TABle 12: The time consumed (in minutes) and the success recognition rate for the different methods using the xm2vts (C2) database.

\begin{tabular}{lcccc}
\hline & PCA & LDA & MPEG-7 & New method \\
\hline Time $(\min )$ & 26.6 & 73.5 & 83.8 & 17.4 \\
Success rate \% & 76 & 92.5 & 93 & 99.9
\end{tabular}

TABle 13: The time consumed (in minutes) and the success recognition rate for the different methods using the FERET $(\mathrm{Fb})$ database.

\begin{tabular}{lcccc}
\hline & PCA & LDA & MPEG-7 & New method \\
\hline Time $(\min )$ & 24.4 & 57.7 & 63.1 & 15.6 \\
Success rate $\%$ & 84 & 92 & 94 & 98.9 \\
\hline
\end{tabular}

TABle 14: The time consumed (in minutes) and the success recognition rate for the different methods using the Yale database.

\begin{tabular}{lcccc}
\hline & PCA & LDA & MPEG-7 & New method \\
\hline Time $(\mathrm{min})$ & 2.8 & 5.1 & 6.3 & 1.6 \\
Success rate $\%$ & 75 & 92.5 & 93 & 96.3 \\
\hline
\end{tabular}

Increasing the number of components has increased the overall performance of the system. Tables 5, 6, 7, 8, 9 show the computational time and performance results of the new method for the various components schemes for the different databases, when $2.8 \mathrm{GHz}-512 \mathrm{k}$ RAM machine is used. The specification of this processor and memory is of quite resemblance to the ones which exist nowadays in our daily used portable devices. Hence, the obtained results are of a great importance as they reflect practical situations.

The results obtained in Tables 5, 6, 7, 8, 9 reveal the following. The overall performance of the system is steady with the different databases. Another observation that can be made from these tables (columns 1, 2, and 3) is that the success rate is stable even by decreasing the component size from $30 \times 30$ to $20 \times 20$ (with the number of components kept unchanged, 4 components in this case). However, the processing time has reduced. Also, fixing the component size to $20 \times 20$ (columns $3,4,5,6$, and 7) and increasing the number of components led to an increase in the success rate. Further, comparing Tables 6 and 7 for the xm2vts database 
under the two configurations, we see that in general the performance of our method was better on (C2) scheme. This could be explained by the fact that the evaluation data available for setting the operational thresholds are more representatives, as it was recorded in a completely different session. Moreover, we notice that the variation in illumination and large poses has affected the results of Table 9, when compared to other databases results. Further, the results show that in general 14 -component of size $20 \times 20$ gives optimal results when the two factors are taken into consideration: the performance and the least possible time consumption. For example, the $30 \times 30$ four-component achieves $99.7 \%$ success rate on the xm2vts (C2) database, but it takes almost 21 minutes to accomplish its task. On the other hand, the $20 \times 2014$-components achieves $99.9 \%$ success rate in almost 17 minutes. Finally, it should be noted that the performance of the system has saturated at this high level when the number of components was increased to more than 14 (but the processing time has increased more).

To take a closer look at the effect of component size on system's complexity, we provide a plot of computation time as a function of block (component) size. Figure 5 shows the time for a single block of size $45 \times 45$ down to size $10 \times 10$ for the xm2vts (C1) database case. As seen from the plot, the processing time drops dramatically as the window size decreases to $35 \times 35$. Then, it starts to decrease at a slower rate until the size of the image reaches $20 \times 20$. Note that processing a single component of size $25 \times 25$ takes around 4 minutes whereas the processing time for a single component of size $20 \times 20$ takes less than 2 minutes.

Also, we present the performance statistics and report them as cumulative match scores (CMS). In this case, identification is regarded as correct if the true object is in the top rank $n$ matches. The results for the ORL, FERET $(\mathrm{Fb})$, and Yale databases are shown in Figures 6, 7, and 8 , respectively. The result for the new method is given for the case of 14 components size $20 \times 20$. Also, it is worth mentioning that these curves were calculated for the MPEG7, Holistic LDA, and PCA [61] techniques as well, as shown in the figures.

Furthermore, we provide additional comparisons with other methods which include the computational time consumed by each one of them. Tables 10, 11, 12, 13, 14 show the computational time and performance results of the new method against the MPEG-7, Holistic LDA, and PCA, for the different databases. Again, the result for the new method is given for the case of 14 components size $20 \times 20$. The obtained results clearly demonstrate the stability and superiority of the new system when compared to the others.

\section{Concluding Remarks}

Due to recently fast advances in technology, face recognition systems that utilize limited memory devices are worth more investigation. In this paper, a new technique which combines the advantages of frequency domain, componentbased approach, SFFS search algorithm, and LDA technique was proposed. The new technique makes the implementation of face recognition on limited memory devices feasible without the fear of deleterious effect on recognition rate. Such implementation would save a considerable amount of computation time and storage requirements due to many reasons. The first reason is that it uses the phase part of the Fourier transform of the face images components, which offers two advantages: (i) minimizing the parameters that negatively affect the efficiency of the system and (ii) saving memory space that is needed when magnitude is present. The other reason is that it is dealing with components of the original image (instead of the whole image), where the calculation of the different scatter matrices does not require large size of memory and hence, the system will not allocate large memory blocks for such calculations. The proposed technique achieved a better performance when compared to other well-known published methods.

\section{References}

[1] W. Zhao, R. Chellappa, P. J. Phillips, and A. Rosenfeld, "Face recognition: a literature survey," ACM Computing Surveys, vol. 35, no. 4, pp. 399-458, 2003.

[2] A. K. Jain, A. Ross, and S. Prabhakar, "An introduction to biometric recognition," IEEE Transactions on Circuits and Systems for Video Technology, vol. 14, no. 1, pp. 4-20, 2004.

[3] K. Lee and H. Byun, "A new face authentication system for memory-constrained devices," IEEE Transactions on Consumer Electronics, vol. 49, no. 4, pp. 1214-1222, 2003.

[4] C. Ng, M. Savvides, and P. K. Khosla, "Real-time face verification system on a cell-phone using advanced correlation filters," in Proceedings of the 4th IEEE Workshop on Automatic Identification Advanced Technologies (AUTO ID '05), pp. 5762, New York, NY, USA, October 2005.

[5] N. Zaeri, F. Mokhtarian, and A. Cherri, "Fast face recognition technique for small and portable devices," in Proceedings of the IEEE International Workshop on Imagining Systems and Techniques (IST '06), pp. 114-118, Sorrento, Italy, April 2006.

[6] P. Wang, L. Tran, and Q. Ji, "Improving face recognition by online image alignment," in Proceeding of the 18th IEEE Computer Society International Conference on Pattern Recognition (ICPR '06), vol. 1, pp. 311-314, 2006.

[7] N. Zaeri, F. Mokhtarian, and A. Cherri, "Efficient face recognition for wireless surveillance systems," in Proceedings of the 9th IASTED International Conference on Computer Graphics and Imaging (CGIM '07), pp. 132-137, Innsbruck, Austria, February 2007.

[8] G. Aggarwal, A. K. Roy-Chowdhury, and R. Chellappa, "A system identification approach for video-based face recognition," in Proceedings of the 17th International Conference on Pattern Recognition (ICPR '04), vol. 4, pp. 175-178, August 2004.

[9] N. Zaeri, M. Farzin, and A. Cherri, "Binarized eigenphases applied to limited memory face recognition systems," Pattern Analysis and Applications, vol. 11, no. 3-4, pp. 373-383, 2008.

[10] B. S. Manjunath, P. Salembier, and T. Sikora, Introduction to MPEG-7, John Wiley \& Sons, 2002.

[11] N. Zaeri, "Memory efficient face recognition using compressed phase component," in Proceedings of the 51st International Symposium (ELMAR '09), pp. 41-44, Zadar, Croatia, September 2009.

[12] T. K. Kim, H. Kim, W. Hwang, and J. Kittler, "Componentbased LDA face description for image retrieval and MPEG-7 
standardisation," Image and Vision Computing, vol. 23, no. 7, pp. 631-642, 2005.

[13] S. Mitra and Y. Liu, "Local facial asymmetry for expression classification," in Proceedings of IEEE Conference on Computer Vision and Pattern Recognition (CVPR '04), vol. 2, pp. 889-894, Washington, DC, USA, July 2004.

[14] A. Mohan, C. Papageorgiou, and T. Poggio, "Example-based object detection in images by components," IEEE Transactions on Pattern Analysis and Machine Intelligence, vol. 23, no. 4, pp. 349-361, 2001.

[15] S. Ullman, M. Vidal-Naquet, and E. Sali, "Visual features of intermediate complexity and their use in classification," Nature-Neuroscience, vol. 5, no. 7, pp. 682-687, 2002.

[16] G. Dorko and C. Schmid, "Selection of scale invariant neighborhoods for object class recognition," in Proceedings of the 9th IEEE International Conference on Computer Vision, pp. 634-640, October 2003.

[17] B. Heisele, T. Serre, M. Pontil, T. Vetter, and T. Poggio, "Categorization by learning and combining object parts," in Proceedings of the Neural Information Processing Systems (NIPS '01), pp. 1239-1245, 2001.

[18] R. Fergus, P. Perona, and A. Zisserman, "Object class recognition by unsupervised scale-invariant learning," in Proceedings of the IEEE Conference on Computer Vision and Pattern Recognition, pp. 264-271, June 2003.

[19] D. G. Lowe, "Distinctive image features from scale-invariant keypoints," International Journal of Computer Vision, vol. 60, no. 2, pp. 91-110, 2004.

[20] C. Kim, J. Oh, and C. Choi, "Combined subspace method using global and local features for face recognition," in Proceedings of the IEEE International Joint Conference on Neural Networks (IJCNN'05), vol. 4, pp. 2030-2035, Montreal, Canada, 2005.

[21] P. Viola and M. Jones, "Rapid object detection using a boosted cascade of simple features," in Proceedings of the IEEE Computer Society Conference on Computer Vision and Pattern Recognition (CVPR '01), vol. 1, pp. 511-518, December 2001.

[22] X. Chen, L. Gu, S. Li, and H.-J. Zhang, "Learning representative local features for face detection," in Proceedings of the IEEE Computer Society Conference on Computer Vision and Pattern Recognition, pp. 1126-1131, December 2001.

[23] Y. Amit and D. Geman, "A computational model for visual selection," Neural Computation, vol. 11, no. 7, pp. 1691-1715, 1999.

[24] B. Heisele and T. Koshizen, "Components for face recognition," in Proceedings of the 6th IEEE International Conference on Automatic Face and Gesture Recognition (FGR '04), pp. 153158, May 2004.

[25] Y. Ivanov, B. Heisele, and T. Serre, "Using component features for face recognition," in Proceedings of the 6th International Conference on Automatic Face and Gesture Recognition (FGR '04), pp. 421-426, May 2004.

[26] A. M. Martinez, "Recognizing imprecisely localized, partially occluded, and expression variant faces from a single sample per class," IEEE Transactions on Pattern Analysis and Machine Intelligence, vol. 24, no. 6, pp. 748-763, 2002.

[27] B. Heisele, T. Serre, and T. Poggio, "A component-based framework for face detection and identification," International Journal of Computer Vision, vol. 74, no. 2, pp. 167-181, 2006.

[28] R. Brunelli and T. Poggio, "Face recognition: features versus templates," IEEE Transactions on Pattern Analysis and Machine Intelligence, vol. 15, no. 10, pp. 1042-1052, 1993.
[29] B. Moghaddam and A. Pentland, "Probabilistic visual learning for object representation," IEEE Transactions on Pattern Analysis and Machine Intelligence, vol. 19, no. 7, pp. 696-710, 1997.

[30] L. Wiskott, J.-M. Fellous, N. Kruger, and C. V. D. von Malsburg, "Face recognition by elastic bunch graph matching," IEEE Transactions on Pattern Analysis and Machine Intelligence, vol. 19, no. 7, pp. 775-779, 1997.

[31] K. Tan and S. Chen, "Adaptively weighted sub-pattern PCA for face recognition," Neurocomputing, vol. 64, no. 1-4, pp. 505511, 2005.

[32] J. Short, J. Kittler, and K. Messer, "Photometric normalisation for component-based face verification," in Proceedings of the 7th International Conference on Automatic Face and Gesture Recognition (FGR '06), pp. 114-119, Southampton, UK, April 2006.

[33] W.-Y. Lin, M.-Y. Chen, K. R. Widder, Y. H. Hu, and N. Boston, "Fusion of multiple facial regions for expression-invariant face recognition," in Proceedings of the IEEE 9th International Workshop on Multimedia Signal Processing (MMSP '07), pp. 426-429, Crete, Greece, October 2007.

[34] P. Tsai, T. Jan, and T. Hintz, "Kernel-based subspace analysis for face recognition," in Proceedings of the International Joint Conference on Neural Networks (IJCNN '07), pp. 1127-1132, Orlando, FL, USA, August 2007.

[35] B. Zhang, S. Shan, X. Chen, and W. Gao, "Histogram of Gabor phase patterns (HGPP): a novel object representation approach for face recognition," IEEE Transactions on Image Processing, vol. 16, no. 1, pp. 57-68, 2007.

[36] J. Zou, Q. Ji, and G. Nagy, "A comparative study of local matching approach for face recognition," IEEE Transactions on Image Processing, vol. 16, no. 10, pp. 2617-2628, 2007.

[37] Z. Tongzhou, L. Jin, A. Zelin, C. Nian, and M. Hui, "New image fusion-based algorithm to face recognition," in Proceedings of the 3rd International Conference on Multimedia and Ubiquitous Engineering (MUE '09), pp. 105-108, Qingdao, China, June 2009.

[38] S. Du and R. Ward, "Improved face representation by nonuniform multilevel selection of gabor convolution features," IEEE Transactions on Systems, Man, and Cybernetics-Part B, vol. 39, no. 6, pp. 1408-1419, 2009.

[39] R. Kam-Art, T. Raicharoen, and V. Khera, "Face recognition using feature extraction based on descriptive statistics of a face image," in Proceedings of the 8th International Conference on Machine Learning and Cybernetics, pp. 193-196, Baoding, China, July 2009.

[40] B. Hua and T. Liu, "Facial expression recognition based on local feature bidirectional 2DPCA," in Proceedings of the International Conference on Information Technology and Computer Science (ITCS '09), pp. 301-304, Kiev Kyiv, Ukraine, July 2009.

[41] M. De Marsico, M. Nappi, and D. Riccio, "FARO: face recognition against occlusions and expression variations," IEEE Transactions on Systems, Man, and Cybernetics—Part A, vol. 40, no. 1, pp. 121-132, 2010.

[42] J. Harguess and J. K. Aggarwal, "A case for the average-halfface in 2D and 3D for face recognition," in Proceedings of the IEEE Computer Society Conference on Computer Vision and Pattern Recognition (CVPR '09), pp. 7-12, Miami, FL, USA, June 2009.

[43] N. Zaeri, J. Kittler, and A. Cherri, "Efficient component-based face recognition system for high speed devices," in Proceedings of the International Conference on Design and Architectures for Signal and Image Processing, Brussels, Belgium, 2008. 
[44] Y. Choi, T. Tokumoto, M. Lee, and S. Ozawa, "Incremental two-dimensional two-directional principal component analysis (I(2D) ${ }^{2}$ PCA) for face recognition," in Proceedings of the IEEE International Conference on Acoustics, Speech and Signal Processing (ICASSP '11), 2011.

[45] R. Atta and M. Ghanbari, "Low-memory requirement and efficient face recognition system based on DCT pyramid," IEEE Transactions on Consumer Electronics, vol. 56, no. 3, pp. 1542-1548, 2010.

[46] C. He, C. Sui, J. Chen, and C. Xixin, "Face-recognition hardware implementation based on SOPC," in Proceedings of the 12th International Conference on Advanced Communication Technology (ICACT '10), pp. 1595-1599, Phoenix Park, Ireland, February 2010.

[47] D. Sisodia, S. K. Shrivastava, and R. C. Jain, "ISVM for face recognition," in Proceedings of the International Conference on Computational Intelligence and Communication Networks (CICN '10), pp. 554-559, Bhopal, India, November 2010.

[48] K. Xi, Y. Tang, J. Hu, and F. Han, "A correlation based face verification scheme designed for mobile device access control: from algorithm to java ME implementation," in Proceedings of the 5th IEEE Conference on Industrial Electronics and Applications (ICIEA '10), pp. 317-322, Taichung, Taiwan, June 2010.

[49] E. Vazquez-Fernandez, H. Garcia-Pardo, D. GonzalezJimenez, and L. Perez-Freire, "Built-in face recognition for smart photo sharing in mobile devices," in Proceedings of the IEEE International Conference on Multimedia and Expo (ICME '11), pp. 1-4, Barcelona, Spain, July 2011.

[50] Y.-C. Wang and K.-T. Cheng, "Energy-optimized mapping of application to smartphone platform - acase study of mobile face recognition," in Proceedings of the IEEE Computer Society Conference on Computer Vision and Pattern Recognition Workshops, 2011.

[51] A. V. Oppenheim and J. S. Lim, "Importance of phase in signals," Proceedings of the IEEE, vol. 69, no. 5, pp. 529-541, 1981.

[52] M. Savvides, B. V. K. Kumar, and P. K. Khosla, "Eigenphases vs. eigenfaces," in Proceedings of the 17th International Conference on Pattern Recognition (ICPR '04), pp. 810-813, IEEE, August 2004.

[53] K. Etemad and R. Chellappa, "Discriminant analysis for recognition of human face images," Journal of the Optical Society of America A, vol. 14, no. 8, pp. 1724-1733, 1997.

[54] P. N. Belhumeur, J. P. Hespanha, and D. J. Kriegman, "Eigenfaces vs. fisherfaces: recognition using class specific linear projection," IEEE Transactions on Pattern Analysis and Machine Intelligence, vol. 19, no. 7, pp. 711-720, 1997.

[55] R. O. Duda, P. E. Hart, and D. G. Stork, Pattern Classification, John Wily \& Son, New York, NY, USA, 2nd edition, 2000.

[56] M.-H. Yang, "Kernel eigenfaces vs. kernel fisherfaces: face recognition using kernel methods," in Proceedings of the 5th IEEE International Conference on Automatic Face and Gesture Recognition, pp. 215-220, Washington, DC, USA, May 2002.

[57] W.-S. Zheng, J.-H. Lai, and P. C. Yuen, "GA-fisher: a new LDA-based face recognition algorithm with selection of principal components," IEEE Transactions on Systems, Man, and Cybernetics - Part B, vol. 35, no. 5, pp. 1065-1078, 2005.

[58] J. Lu, K. N. Plataniotis, and A. N. Venetsanopoulos, "Face recognition using kernel direct discriminant analysis algorithms," IEEE Transactions on Neural Networks, vol. 14, no. 1, pp. 117-126, 2003.
[59] E. Cantú-Paz, S. Newsam, and C. Kamath, "Feature selection in scientific applications," in Proceedings of the 10th ACM International Conference on Knowledge Discovery and Data Mining (KDD '04), pp. 788-793, August 2004.

[60] K. Messer, J. Matas, J. Kittler, J. Luettin, and G. Maitre, "XM2VTSDB: the extended M2VTS database," in Proceedings of the 2nd International Conference on Audio and Video-based Biometric Person Authentication (AVBPA '99), Washington, DC, USA, 1999.

[61] M. Turk and A. Pentland, "Eigenfaces for recognition,” Journal of Cognitive Neuroscience, vol. 3, no. 1, pp. 71-86, 1991. 

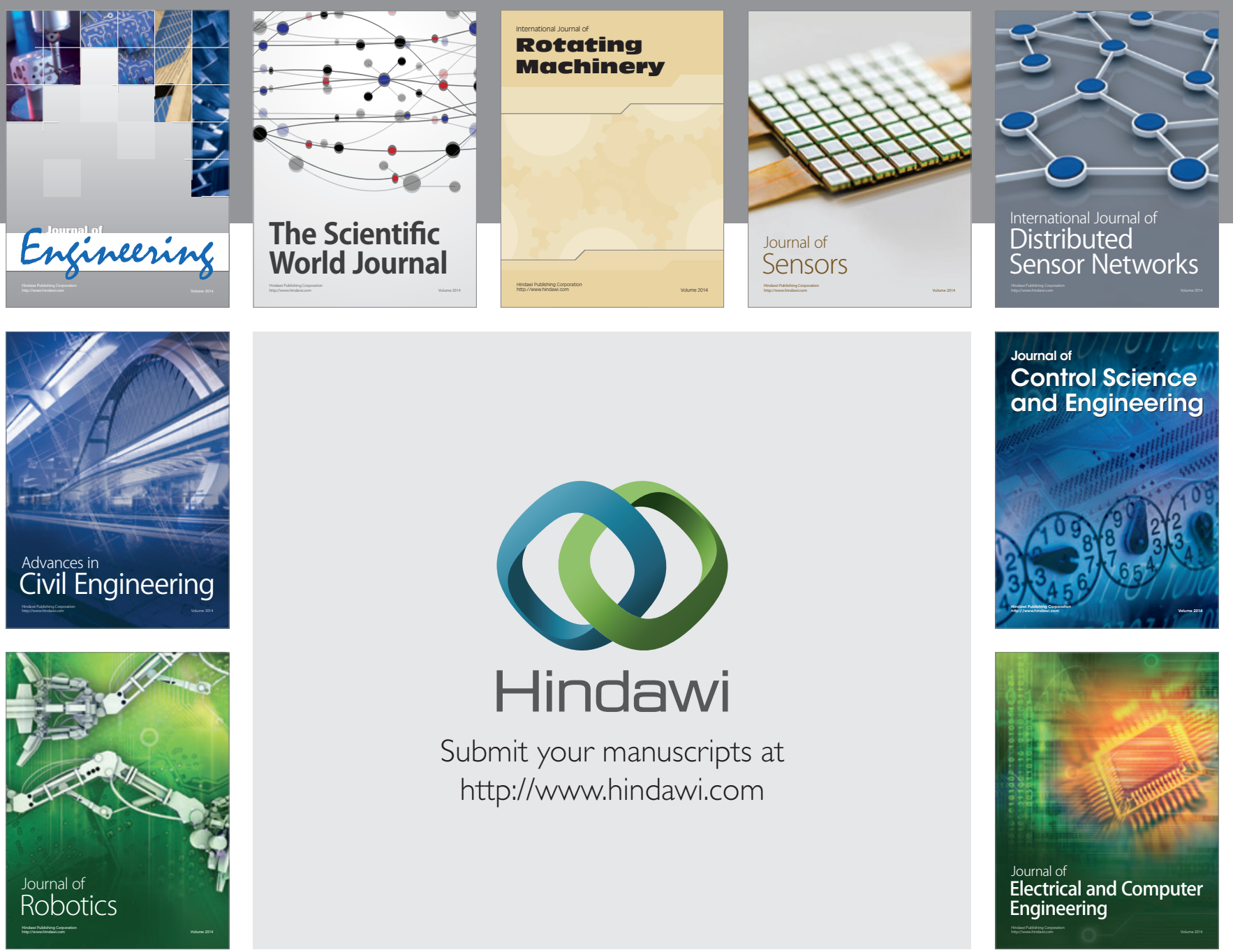

Submit your manuscripts at

http://www.hindawi.com
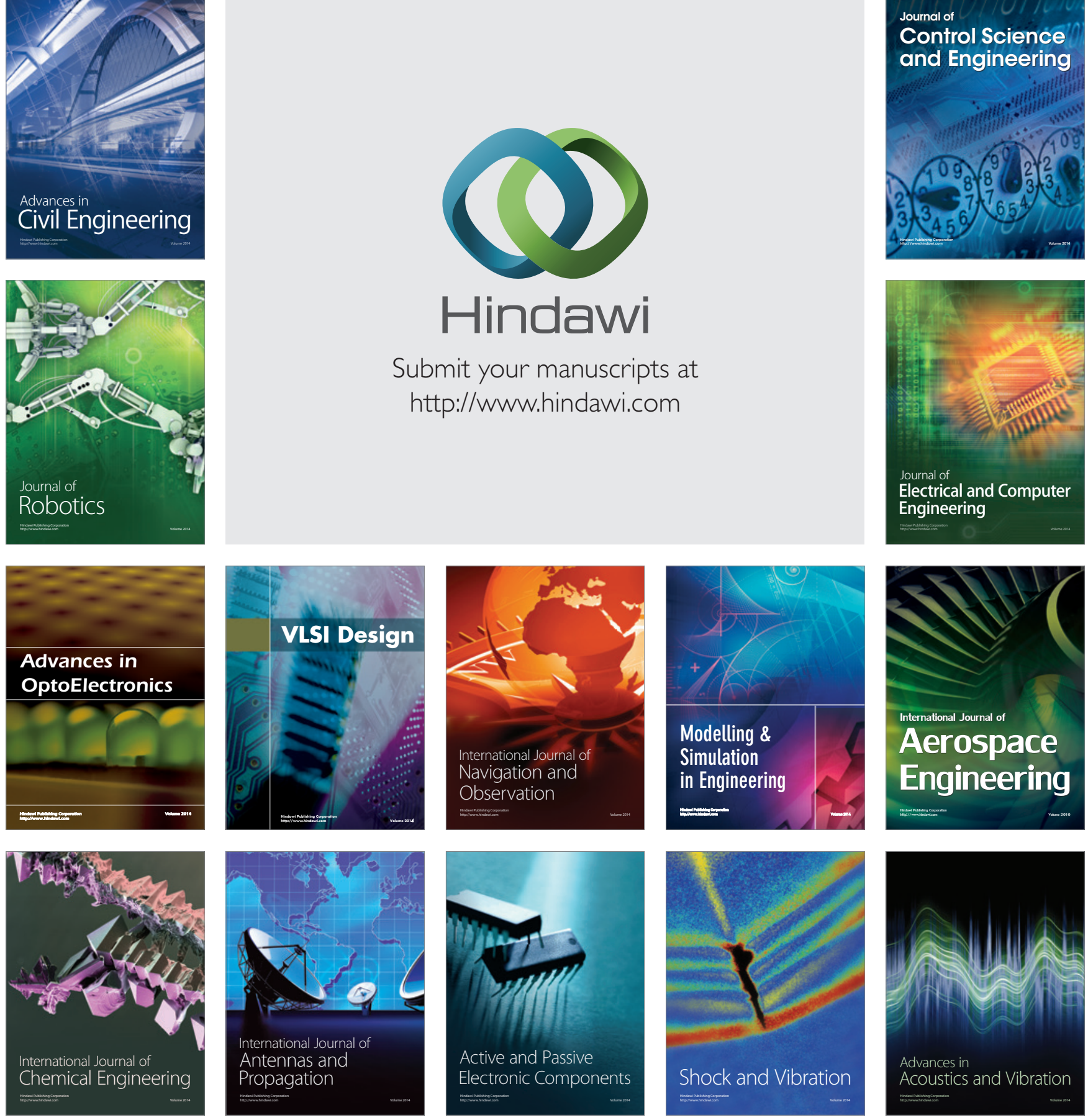